\title{
FPGA implementation of Reed-Solomon Codes Based on Visible Light Wireless Communication System
}

\author{
Lipeng Qiü, a , Xiaopeng Chen ${ }^{1, b}$, Lin Zhou ${ }^{1,2, c}$ and Yucheng He $\mathrm{H}^{1,2, \mathrm{~d}}$ \\ ${ }^{1}$ The Xiamen Key Laboratory of Mobile Multimedia Communications, \\ National Huaqiao University, Xiamen, China \\ 2 The State Key Laboratory of Integrated Services Networks, \\ Xidian University, Xi'an, China \\ a347885526@qq.com, b xiaopeng2122@sina.com, \\ clinzhou@hqu.edu.cn, dyucheng.he@hqu.edu.cn
}

\begin{abstract}
Keywords: Reed-Solomon codes; VLC; RiBM algorithm; FPGA.
Abstract. The research of visible light communications $(V L C)$ has became a focus of wireless communications in recent years. In this paper, the channel coding technique for $V L C$ systems were studied. According to the characteristics of VLC channel, RS codes were designed to correct the burst and random errors. The pipeline architecture was designed in the proposed system, and the RS decoder was divided into 4 modules, the syndrome calculation module, the key equation calculation module, the Chein Search module and the Forney algorithm module. In the Chein search module, the RiBM algorithm was used for reduction of complexity. All FPGA implementations of the 4 modules were provided in detail, and simulation results were shown on the Quartus II 9.0 platform. The simulation results show that, no more than 8 random and burst errors can be completely correct by one single proposed RS $(204,188)$ codeword.
\end{abstract}

\section{Introduction}

Visible light communication can transmit wireless information by using fast light pulse, such as coded '1' when the LED is on, otherwise coded ' 0 '. VLC technology can be instead of conventional wireless communications in some places with forbidden of the electromagnetic interferences, such as airport,hospital etc. In a practical communication system, channel coding technology can provide a guaranteed coding gain, which can save the transmission power and increase the distance of communications.

Recently, in VLC channel analysis, system designing, modulation and signal detection have attracted much attention. As known, the burst errors occurred frequently in VLC system because of many indoor factors, like occlusion, fading and noise. In this paper, first we chose a RS code from Digital Vedio Broadcasting (DVB) standard for our VLC scheme in order to improve the quality of communications. Then the implementations of the RS encoder and decoder were proposed in detail, and some simulation results were presented to prove the effectiveness. At last, the experiments showed that the RS-based VLC system worked well on the FPGA Cyclone IV EP4CE15F17C8N.

\section{Visible Light Communication System}

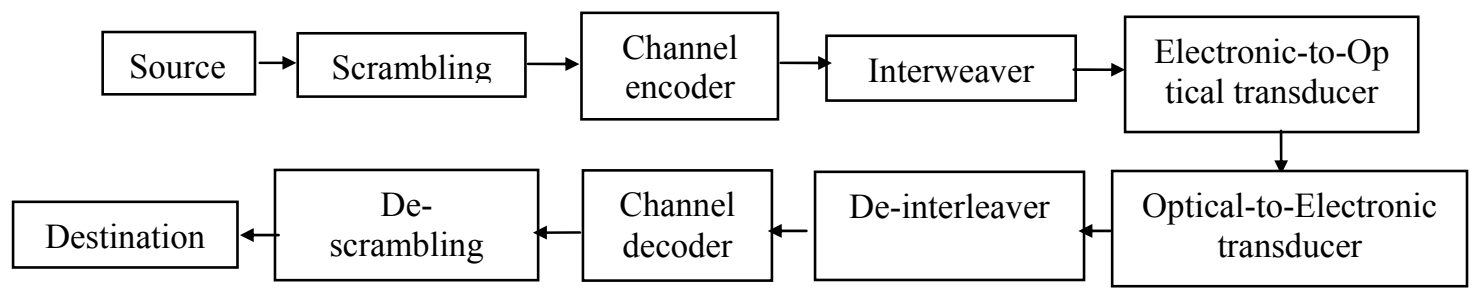

Fig. 1 VLC system process diagram

The diagram of VLC system is shown in Fig. 1. The optical transmitter consists of source, scrambling, channel encoder, interleaver and electronic-to-optical transducer. The information bits should be 
processed before they transmitted. For example, the scrambling module eliminates continuous ' 0 ' or ' 1 ' in source information, the channel encoder module reduces BER during transmission, the interleaver module avoids the burst errors occurred. The electronic-to-optical transducer transfers electronic signal into optical signal. Correspondingly, the optical receiver is made up of optical-to-electronic transducer, de-interleaver, channel decoder, de-scrambing and destination. Receiver restores the processed information in correctly.

\section{Brief Introduction of RS Codes}

RS Encode. Reed-Solomon (RS) code is a class of linear block codes, see [1]. So RS codes can be explained as non-binary $\mathrm{BCH}$ codes. The values of code coefficient are taken from $\mathrm{GF}\left(2^{\mathrm{m}}\right)$, refer to [2]. Let $\alpha$ be the primitive element of $\mathrm{GF}\left(2^{\mathrm{m}}\right)$. For a t-error correcting RS codes, the roots of generator polynomial $\mathrm{g}(\mathrm{x})$ are $\alpha, \alpha^{2}, \ldots, \alpha^{2 \mathrm{t}}$,and

$$
\mathrm{g}(\mathrm{x})=(\mathrm{x}-\alpha)\left(\mathrm{x}-\alpha^{2}\right) \cdots\left(\mathrm{x}-\alpha^{2 \mathrm{t}}\right)=\mathrm{g}_{0}+\mathrm{g}_{1} \cdot \mathrm{x}+\cdots+\mathrm{g}_{2 \mathrm{t}-1} \cdot \mathrm{x}^{2 \mathrm{t}-1}+\mathrm{x}^{2 \mathrm{t}} \text {. }
$$

Where $\mathrm{g}_{0}, \mathrm{~g}_{1}, \ldots, \mathrm{g}_{2 \mathrm{t}-1}$ are the coefficient of generator polynomial.

If un-coded information polynomial is

$$
\mathrm{m}(\mathrm{x})=\mathrm{m}_{0}+\mathrm{m}_{1} \cdot \mathrm{x}+\cdots+\mathrm{m}_{\mathrm{k}-1} \cdot \mathrm{x}^{\mathrm{k}-1} \text {. }
$$

Check polynomial

$$
\mathrm{r}(\mathrm{x})=\mathrm{r}_{0}+\mathrm{r}_{1} \cdot \mathrm{x}+\cdots+\mathrm{r}_{\mathrm{n}-\mathrm{k}-1} \cdot \mathrm{x}^{\mathrm{n}-\mathrm{k}-1} \text {. }
$$

Where $k$ is the number of information bits, $n$ is code length.

Thus we can get code polynomial

$$
\mathrm{c}(\mathrm{x})=\mathrm{x}^{2 \mathrm{t}} \mathrm{m}(\mathrm{x}) \bmod \mathrm{g}(\mathrm{x}) \text {. }
$$

The design of RS codes encoder is shown in Fig. 2.

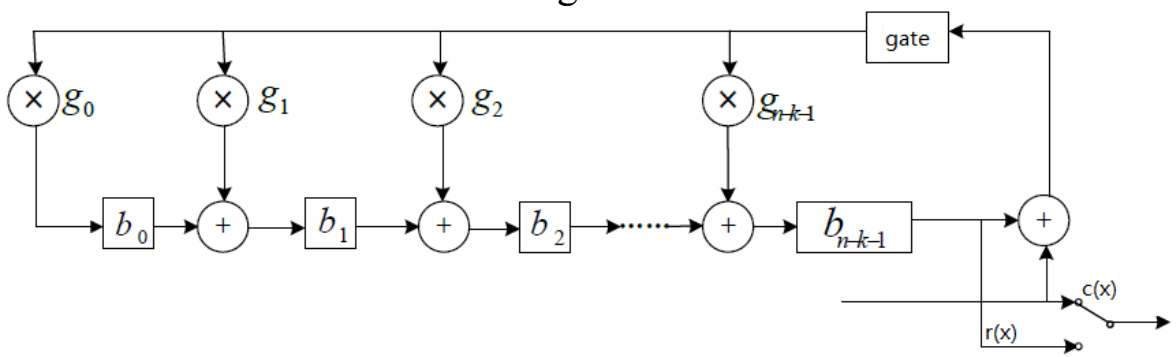

Fig. 2 RS codes encoder schematic diagram

RS Decode. When decoder receive the signal $\mathrm{R}(x)$ from channel, it need to detect and correct errors in $\mathrm{R}(x)$. The processing of decode as follows: First, receiving $\mathrm{R}(x)$ and get syndrome $\mathrm{S}_{i}$ by equation (5). Secondly, according to $\mathrm{S}_{i}$, using BM algorithm given in[3] to get error position polynomial $\Lambda(x)$ and error value polynomial $\Omega(x)$. Thirdly, using Chein Search, given in [4], to detect the positions of errors. Forth, calculating errors' value by Forney equation in [5].Finally, synthesizing errors' position and value. Then we can get the corrected result $c^{6}(x)$.If $c^{6}(x)=c(x)$, decode succeed,or fail. The schematic design of decoder is shown in Fig. 3.

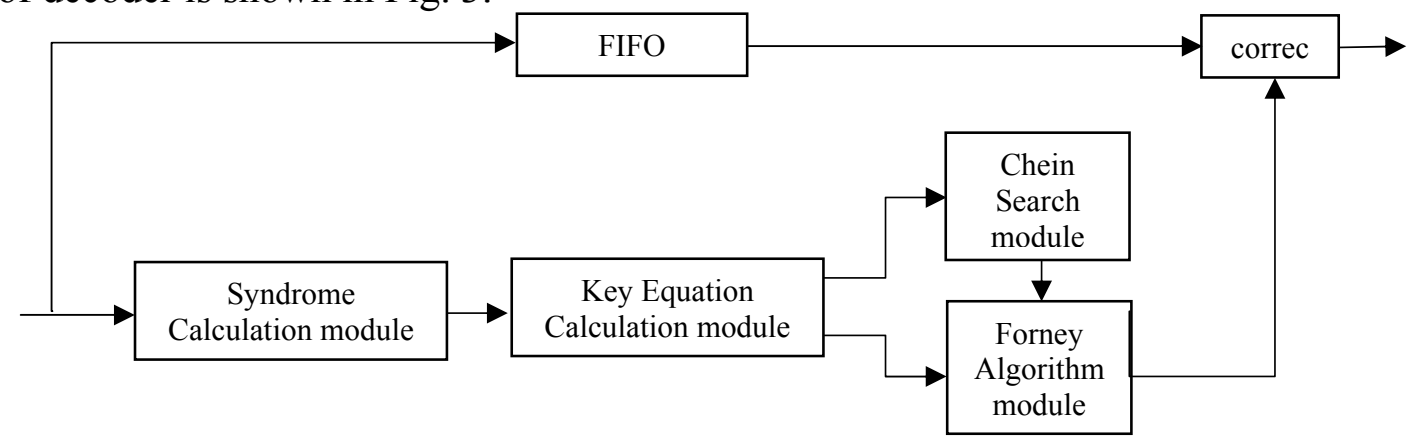

Fig. 3 RS codes decoder schematic diagram 


\section{Main Module Design and Simulation}

At the decoder side, the syndrome calculation (SC) module can be implement by $\operatorname{GF}\left(2^{8}\right)$ multiplier with constant coefficients and shifting register, as indicated in [6]. Calculation formulas is

$$
\mathrm{S}_{\mathrm{i}}=\mathrm{R}\left(\alpha^{\mathrm{i}}\right)=\sum_{\mathrm{j}} \mathrm{r}_{\mathrm{j}} \cdot \alpha^{\mathrm{ij}}, \mathrm{i}=0,1,2, \ldots, 2 \mathrm{t}-1, \mathrm{j}=0,1, \ldots, \mathrm{n}-1 \text {. }
$$

The structure of SC module is shown in Fig. 4.

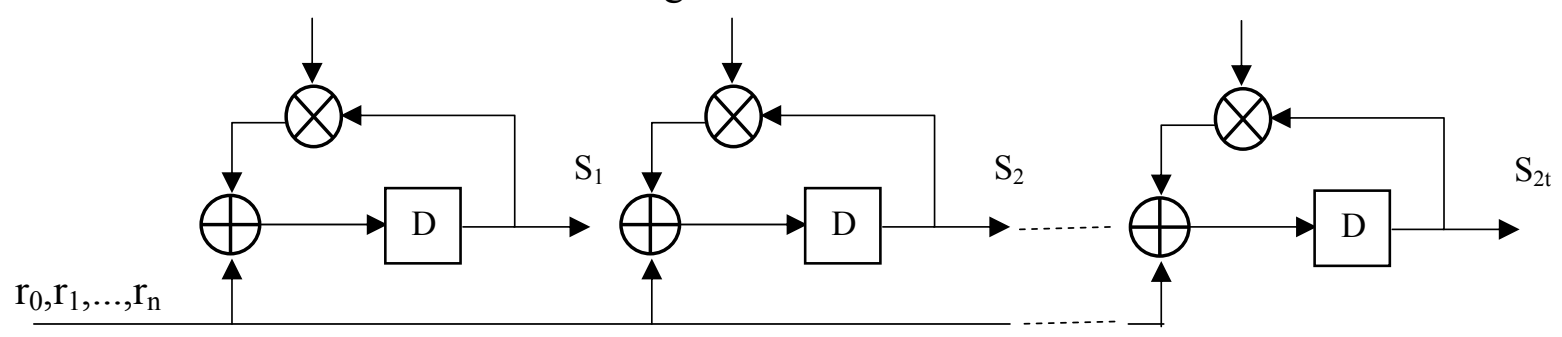

Fig. 4 SC module schematic diagram

The key equation calculation (KEC) module is the key of RS codes decoder, it determines the performance of the decoder. Moreover, this module is the most complicate and time-consuming section. Thus we choose BM algorithm. Because it has shorter iterative path. Usually, we need use RiBM algorithm to eliminate the inverse operation in the algorithm, explained in [7].This algorithm has regular structure and low hardware complexity.

The Chein search (CS) method was proposed in 1964.It is a practical method to search the root of error position polynomial. The structure of Chein search method is shown in Fig. 5.

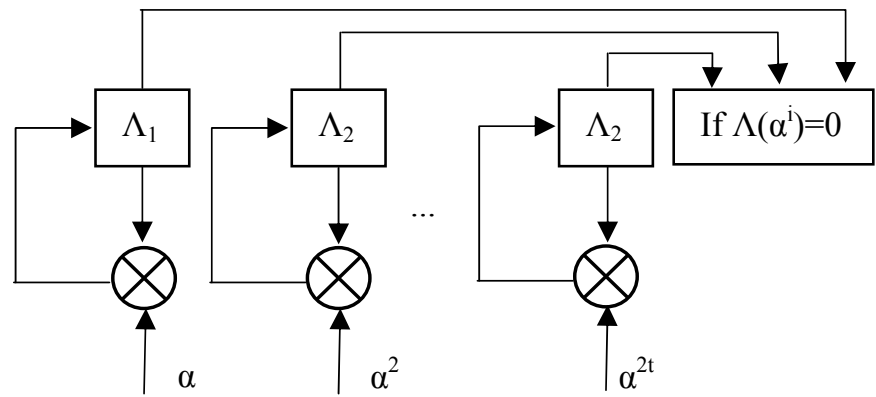

Fig. 5 CS module schematic diagram

The error value is calculated by Forney equation, see [5].

This VLC system is used to simulate source information which has been scrambled, encoded and interleaved, then sent it to transmitter and then reach to an optical receiver over AWGN channel. The decoder is based on RS $(204,188)$ code, with rate 0.92 , the error correcting capability equal to 8 .The VLC system simulation based on soft QUARTUS II 9.0. The simulation of main modules is shown in Fig. 6:

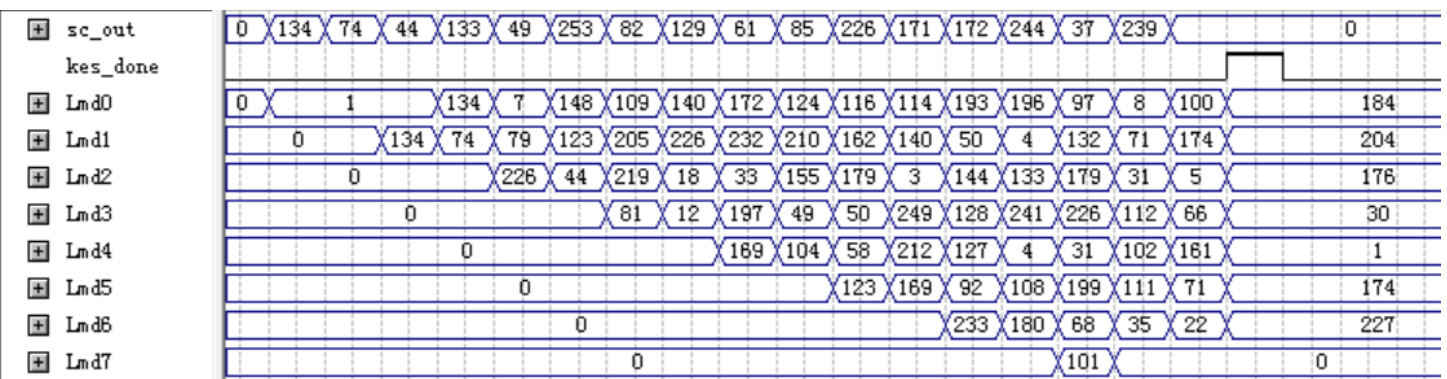

Fig. 6 KEC module simulation result

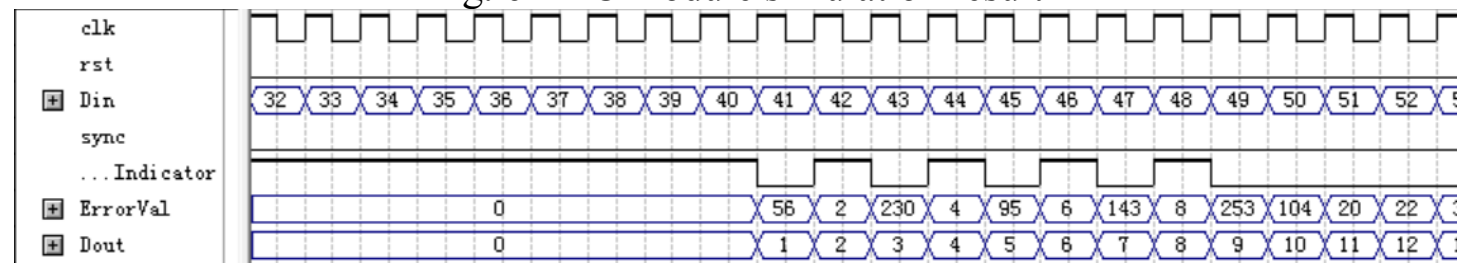

Fig. 7 Chein Search and Forney algorithm simulation result 
The results is shown in Fig. 7. Pin Err_Indicator shows four position of errors and pin ErrorVal displays the corresponding values of four positions. The decoder output (Dout) is equal to input (Din) as we can see from Fig. 7. It shows that the RS codes decoder can apply in VLC system.

\section{Summary}

In this paper, a channel coding scheme for Visible light communications was proposed. We designed and implemented the encoder and decoder of RS $(204,188)$ to anti the burst errors. The decoder was designed with pipeline structure and multipliers over $\operatorname{GF}\left(2^{8}\right)$, which could occupy less hardware resources on FPGA and yield a significant throughput improvement. In future work, if the LDPC codes were applied as the inner codes of the serially concatenated scheme to deal with random errors and the RS codes were applied as the outer codes to anti the burst channel loss, the VLC system performance would be improved better.

\section{Acknowledgements}

This work was supported in part by the grants from the National Natural Science Foundation of China (61302095, 61401165), the Natural Science Foundation of Fujian Province of China (2014J05076, 2014J01243, 2015J01262) and the Science Foundation of National Huaqiao University (12BS219, 13Y0384, 13BS101).

\section{References}

[1] Gallager R G. Low-Density-Parity Check Codes[D]. Cambridge, MA: MIT Press,1963.

[2] Reed I. S. ,Solomon G. Polynomial Codes over certain Finite Fields, Soc. Ind. Appl. Math. , 8:300-304, 1960.

[3] J. L. Massey, "Shift-register Synthesis and BCH Decoding," IEEE Trans. Inform. Theory, 15(1):122-127, 1969.

[4] Qiyue Zhu, “A algorithm of Reed-solomon codes encode and decode," Telecommunication Engineering, 39(2):63-67,1999.

[5] R. E. Blahut. Theory and Practice of Error Control Codes. Addison- Wesley, Reading, Massachusetts, 1983.

[6] Reed I. S. A class of multiple-error-correcting codes and the decoding scheme[J]. Information Theory, Transactions of the IRE Professional Group on, 1954, 4(4):38 - 49.

[7] Glavac V. A VHDL code generator for reed-solomon encoders and decoders [D]. Montreal: Concordia University, 2003.

[8] Sarwate D.V.,Shanbhag N. R. High-speed architectures for Reed-Solomon Decoders[J]. IEEE Trans. on VISI Systems, 2001, 9(5):641-655.

[9] H. M. Shao, T. K. Truong, L. J. Deutsch, J. H. Yuen, and I. S. Reed, “A VLSI design of a pipeline Reed-Solomon decoder,” IEEE Trans. Comput., vol. C-34, pp. 393-403, May 1985. 